\title{
Acceleration of plasma in current sheet during substorm dipolarizations in the Earth's magnetotail: Comparison of different mechanisms
}

Cite as: Phys. Plasmas 26, 042901 (2019); https://doi.org/10.1063/1.5082715

Submitted: 23 November 2018 . Accepted: 07 March 2019 . Published Online: 05 April 2019

Elena I. Parkhomenko, (D) Helmi V. Malova, (D) Elena E. Grigorenko, (D) Victor Yu. Popov, (D) Anatoly A. Petrukovich, (iD) Dominique C. Delcourt, (D) Elena A. Kronberg, (iD) Patrick W. Daly, and (i) Lev M. Zelenyi
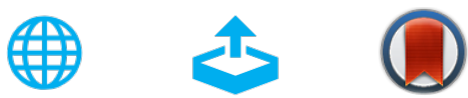

View Online

\section{ARTICLES YOU MAY BE INTERESTED IN}

Whistler wave propagation and interplay between electron inertia and Larmor radius effects Physics of Plasmas 26, 042106 (2019); https://doi.org/10.1063/1.5054374

Fast magnetic reconnection and the ideal evolution of a magnetic field Physics of Plasmas 26, 042104 (2019); https://doi.org/10.1063/1.5081828

Heavy ion acceleration by EMIC waves in the near-Earth plasma sheet

Physics of Plasmas 26, 022903 (2019); https://doi.org/10.1063/1.5075509

\section{AIP Advances Fluids and Plasmas Collection}

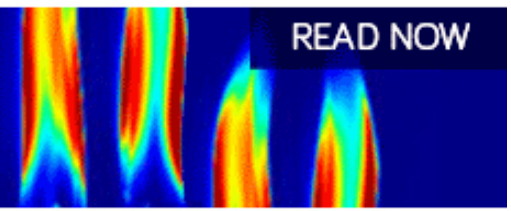




\title{
Acceleration of plasma in current sheet during substorm dipolarizations in the Earth's magnetotail: Comparison of different mechanisms
}

\author{
Cite as: Phys. Plasmas 26, 042901 (2019); doi: 10.1063/1.5082715 \\ Submitted: 23 November 2018 • Accepted: 7 March 2019 • \\ Published Online: 5 April 2019 \\ Elena I. Parkhomenko, ${ }^{7}$ Helmi V. Malova, ${ }^{1,2}$ (D) Elena E. Grigorenko, ${ }^{7}$ (D) Victor Yu. Popov, ${ }^{1,3,4}$ (DD \\ Anatoly A. Petrukovich, ${ }^{7}$ (D) Dominique C. Delcourt, ${ }^{5}$ (D) Elena A. Kronberg, ${ }^{6,7}$ (iD Patrick W. Daly, ${ }^{6}$ \\ and Lev M. Zelenyi ${ }^{1}$ (iD \\ AFFILIATIONS \\ ${ }^{1}$ Space Research Institute of the Russian Academy of Sciences, Moscow 117997, Russia \\ ${ }^{2}$ Scobeltsyn Institute of Nuclear Physics, Lomonosov Moscow State University, Moscow 119991, Russia \\ ${ }^{3}$ Physics Department, Lomonosov Moscow State University, Moscow 119991, Russia \\ ${ }^{4}$ National Research University, "Higher School of Economics," Moscow 101000, Russia \\ ${ }^{5}$ CNRS-Université d'Orléans-CNES, Orléans 45071, France \\ ${ }^{6}$ Max Planck Institute for Solar System Research, Göttingen 37077, Germany \\ ${ }^{7}$ Ludwig-Maximilian University of Munich, Munich 80539, Germany
}

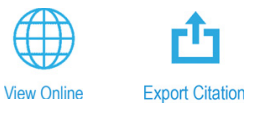

\begin{abstract}
This work is devoted to the investigation of particle acceleration during magnetospheric dipolarizations. A numerical model is presented taking into account the four scenarios of plasma acceleration that can be realized: (A) total dipolarization with characteristic time scales of $\approx 3 \mathrm{~min}$; (B) single peak value of the normal magnetic component $B_{z}$ occurring on the time scale of less than 1 min; (C) a sequence of rapid jumps of $B_{z}$ interpreted as the passage of a chain of multiple dipolarization fronts (DFs); and (D) the simultaneous action of mechanism (C) followed by the consequent enhancement of electric and magnetic fluctuations with the small characteristic time scale $\leq 1 \mathrm{~s}$. In a frame of the model, we have obtained and analyzed the energy spectra of four plasma populations: electrons $\mathrm{e}^{-}$, protons $\mathrm{H}^{+}$, helium $\mathrm{He}^{+}$, and oxygen $\mathrm{O}^{+}$ ions, accelerated by the above-mentioned processes (A)-(D). It is shown that $\mathrm{O}^{+}$ions can be accelerated mainly due to the mechanism (A); $\mathrm{H}^{+}$and $\mathrm{He}^{+}$ions (and to some extent electrons) can be more effectively accelerated due to the mechanism (C) than the single dipolarization (B). It is found that high-frequency electric and magnetic fluctuations accompanying multiple DFs (D) can strongly accelerate electrons $\mathrm{e}^{-}$ and really weakly influence other populations of plasma. The results of modeling demonstrated clearly the distinguishable spatial and temporal resonance character of particle acceleration processes. The maximum particle energies depending on the scale of the magnetic acceleration region and the value of the magnetic field are estimated. The shapes of energy spectra are discussed.
\end{abstract}

https://doi.org/10.1063/1.5082715

\section{INTRODUCTION}

Numerous investigations in the Earth's magnetosphere showed that the processes of plasma acceleration in the magnetotail play an important role in the energy balance of the whole magnetosphere [Sharma et al., 2008; Zelenyi et al., 2008; Retino et al., 2008; Yamada et al., 2010; Fu et al., 2011; Runov et al., 2011; Zelenyi et al., 2011; Angelopoulos et al., 2013; Birn et al., 2012, 2013; Ashour-Abdalla et al., 2015; Grigorenko et al., 2015]. Numerous spacecraft missions (Cluster, THEMIS, MMS, and others) showed that accelerated plasma flows in the Earth's magnetosphere are observed in the periods of the global magnetospheric perturbations as magnetic storms and substorms [Ipavich et al., 1984; Richardson et al., 1987; Delcourt et al., 1990; Lui et al., 1994; Zong et al., 1997; Veltri et al., 1998; Nose et al., 2000; Artemyev et al., 2012; Lui, 2014; Runov et al., 2014; Grigorenko et al., 2015; Kronberg et al., 2017; Liang et al., 2017]. Sometimes, the real acceleration mechanisms cannot be completely interpreted and understood. A series of studies were aimed to understand the origin of particles with energies up to hundreds of $\mathrm{keV}$ in the Earth's magnetotail [e.g., Zhou et al., 2010; Ashour-Abdalla et al., 2015; Kronberg et al., 2017]. The role of transient processes in the magnetotail CS was shown in numerous studies [Baker et al., 1985; Zelenyi et al., 2011; Grigorenko et al., 2011; Birn et al., 2012; Angelopoulos et al., 2013; Lui, 2014].

Indeed, numerous observations reported the presence of transient processes in the magnetotail CS. It was shown that during the 
growth phase of substorm, the CS is thinning to a few ion gyroradii ( $\sim$ a few hundreds of $\mathrm{km}$ ) due to the increase in the magnetic flux in magnetospheric lobes [Sergeev et al., 1993; Sergeev et al., 1996; Runov et al., 2008; Nakamura et al., 2008]. It was shown that such a thin CS is metastable [Galeev, 1979; Baker et al., 1985; Zelenyi et al., 1990; Zelenyi et al., 2008], i.e., it can be stable for quite a long time, but at some moment, it can be explosively destroyed with the consequent magnetic reconnection and the release of a significant value of the reserved magnetic energy.

Frequently, satellites observe TCS near reconnection regions where the particles are accelerated by inductive electric fields. It was shown that these reconnection processes are accompanied by nonlinear moving structures, characterized by rapid jumps of the normal magnetic component $\mathrm{B}_{\mathrm{z}}$ [e.g., Nakamura et al., 2002; Runov et al., 2009; Sergeev et al., 2009], which are named dipolarization fronts (DFs) (here and below, we use the standard solar-magnetospheric coordinate system (GSM), in which the $\mathrm{X}$ axis is directed from the Earth to the Sun, the $\mathrm{Z}$ axis is directed to the north along the geomagnetic dipole, and the $\mathrm{Y}$ axis is directed from the morning to the evening).

DFs can move over large distances from the middle part of the magnetotail toward the Earth without significant evolution [Runov et al., 2011]. Several mechanisms of the formation of DFs have been investigated in works [Sergeev et al., 1992; Heyn and Semenov, 1996; Slavin et al., 2003; Semenov et al., 2005; Longcope and Priest, 2007; Sitnov et al., 2009; Sitnov and Swisdak, 2011]. Initially, the researchers supposed that dipolarization fronts are produced by large-scale X-lines with spatial scales on the order of the magnetotail cross-section [Hoshino et al., 1998, 2000; Baker et al., 2002; Runov et al., 2003; Borg et al., 2005; Wygant et al., 2005; Petkaki et al., 2006; Nakamura et al., 2006]. Recent observations showed that the spatial localization of the DFs propagating in the magnetotail is substantially smaller [Nakamura et al., 2004; Kiehas et al., 2013]. The observations of several spacecrafts have shown that the passage of DFs is followed by strong plasma turbulence and/or electromagnetic fluctuations [Ono et al., 2009; Zhou et al., 2010; Fu et al., 2011; Artemyev et al., 2012; Birn et al., 2012, 2013; El-Alaoui et al., 2013; Lui, 2014; Grigorenko et al., 2016, 2017].

Different hypothetical mechanisms responsible for particle acceleration or heating by DFs have been proposed such as plasma acceleration by the inductive electric field due to the magnetic field reconfiguration [Delcourt et al., 1990; Veltri et al., 1998; Delcourt, 2002; Ono et al., 2009; Greco et al., 2015]. Specifically, the following processes were investigated: (1) the importance of ion nonadiabatic acceleration due to their interaction with the low-frequency magnetic fluctuations in the region behind the dipolarization front [Perri et al., 2009; Greco et al., 2009; Ono et al. 2009]; (2) adiabatic energy gain of magnetized electrons and ions due to the local increase in the magnetic field [Delcourt et al., 1990; Birn et al., 2004; Apatenkov et al., 2007; Fu et al., 2011; Birn et al., 2012]; (3) plasma acceleration near the magnetic reconnection site by the cross-tail electrostatic field $\mathrm{E}_{\mathrm{y}}$ [e.g., Hoshino, 2005; Retino et al., 2008]; (4) particle acceleration in the course of their reflection from fronts [Zhou et al., 2010, 2012]; (5) resonant capture by fronts [Artemyev et al., 2012b; Ukhorskiy et al., 2017]; and (6) particle pick-up in outflows near reconnection regions and consequent heating up to thermal velocities on the order of the Alfvén speed [Drake et al., 2009].Theoretical investigations [e.g.,
Zelenyi et al., 1990; Veltri et al., 1998; Zelenyi et al., 2008; Greco et al., 2009; Artemyev et al., 2012] and recent studies [Greco et al., 2015; Grigorenko et al., 2015; Catapano et al., 2016; Liang et al., 2017] have demonstrated that the spatial resonance between particles and electromagnetic fields depends on particle Larmor radii and the nonstationary magnetic configurations. However, the problem of identification of mechanisms responsible for a strong particle acceleration is not solved yet in general, and the interpretation of a presumable acceleration of a plasma population in the multicomponent plasma often cannot be done.

In this paper, we investigated 4 general scenarios (mechanisms) of plasma acceleration during magnetic dipolarization chosen on the basis of real observations: (A) total dipolarization with characteristic time scales of $\approx 3 \mathrm{~min}$; (B) single peak of the normal magnetic field component $B_{z}$ taking place at time scales $<1 \mathrm{~min}$; (C) a set of sharp $B_{z}$ jumps interpreted as the passage of multiple dipolarization fronts; and (D) the simultaneous action of (C) and enhancement of electric and magnetic fluctuations with the smallest characteristic time scales $(\leq 1 \mathrm{~s})$. These oscillations sometimes are observed at the later stage of dipolarization and can contribute to energization of electrons [Grigorenko et al., 2016]. All considered mechanisms (A)-(D) assume variations of the normal magnetic field $B_{z}$ in the magnetotail current sheet on different temporal scales that can effectively interact with the charged particles and accelerate them. Also, the obtained numerical results for different transient phenomena (A)-(D) associated with dipolarizations are analyzed, and comparison of both average and maximum particle energies has been done. The role of resonant spatial and temporal resonant effects in plasma particle acceleration during substorms is discussed.

\section{THE MODEL}

In our model, the vector of the magnetic field of the base model of the current sheet is taken in the form

$$
\mathbf{B}_{\mathbf{0}}=B_{x 0} \mathbf{e}_{x}+B_{y 0} \mathbf{e}_{y}+B_{z 0} \mathbf{e}_{z} .
$$

Here, the tangential magnetic component $B_{x 0}(z)$ changes its sign in the neutral plane $z=0$, equal to $B_{x}=20 \mathrm{nT}$ at the boundaries of the current sheet, and is described in a frame of the Harris model [Harris, 1962; Lembege and Pellat, 1982]

$$
B_{x 0}=B_{x} \cdot \tanh \left(\frac{z}{L}\right) .
$$

Here, $L$ is the current sheet thickness. Magnetic shear is absent, $B_{y 0}=0$. The value of the normal component is $B_{z 0}=2 \mathrm{nT}$. In the initial state of the system, the large-scale electric field is taken in the form

$$
\mathbf{E}_{0}=E_{x 0} \mathbf{e}_{x}+E_{y 0} \mathbf{e}_{y}+E_{z 0} \mathbf{e}_{z},
$$

where $E_{x 0}=0, E_{y 0}=0.2 \mathrm{mV} / \mathrm{m}$, and $E_{z 0}=0$. Therefore, we take into account the large-scale electric field in the magnetotail persisting in the quiet magnetosphere. Let us note that our model, expression (2), is not the self-consistent solution of current sheet equilibrium. Below, the particle tracing method in prescribed magnetic and electric fields is used to investigate the energy spectra of particles during nonstationary changes of electric and magnetic fields. 
We have considered the particle dynamics in the magnetotail configuration $\mathbf{B}_{0}(z)$ in the presence of a magnetic dipolarization with magnetic jump $\Delta \mathbf{B}(t)$ followed by high frequency fluctuations of electric field $\delta \mathbf{E}(\mathbf{r}, t)$ and corresponding oscillating magnetic field $\delta \mathbf{B}(\mathbf{r}, t)$. The magnetic field can be considered as a superposition of three components

$$
\mathbf{B}(\mathbf{r}, t)=\mathbf{B}_{0}(z)+\Delta \mathbf{B}(\boldsymbol{t})+\delta \mathbf{B}(\mathbf{r}, t),
$$

where $\mathbf{B}_{0}(z)$ is the initial magnetic field of the magnetotail current sheet; $\Delta \mathbf{B}(\boldsymbol{t})=\Delta\left\{\mathbf{B}_{d}(t)+\mathbf{B}_{D f}(t)\right\}$ is the magnetic field of the general dipolarization $\Delta \mathbf{B}_{\mathrm{Df}}(t)$ and multiple dipolarization fronts $\Delta \mathbf{B}_{\mathrm{d}}(t)$ coming to the observer at the moment of time $t$; and $\delta \mathbf{B}(\mathbf{r}, t)$ is the magnetic field of high-frequency turbulence.

The corresponding electric field is taken in the form

$$
\mathbf{E}(\mathbf{r}, t)=\mathbf{E}_{0}+\Delta \mathbf{E}(\mathbf{r}, t)+\delta \mathbf{E}(\mathbf{r}, t)
$$

where $\mathbf{E}_{0}$ is the initial large-scale electric field; $\Delta \mathbf{E}(\mathbf{r}, \boldsymbol{t})=\Delta\left\{\mathbf{E}_{d}(\mathbf{r}, t)\right.$ $\left.+\mathbf{E}_{D f}(\mathbf{r}, t)\right\}$ is the inductive electric field consisting of the dipolarization trend $\mathbf{E}_{\mathrm{Df}}(\mathbf{r}, t)$ and frequent multiple dipolarization fronts $\mathbf{E}_{\mathrm{d}}(\mathbf{r}, t)$ that were described using Maxwell equations

$$
\begin{aligned}
\nabla \times \Delta \mathbf{E} & =-\frac{1}{\mathrm{c}} \frac{\partial \Delta \mathbf{B}}{\partial t}, \\
\nabla \cdot \Delta \mathbf{E} & =0 .
\end{aligned}
$$

The term $\delta \mathbf{E}(\mathbf{r}, t)$ is the electric field of high-frequency turbulence which was observed during Cluster observations on July 20, 2013 (from 01:33:08 to 01:48:11 UT) and was presented as the ensemble of plane waves $\delta \mathbf{E}(\mathbf{r}, t)$

$$
\begin{aligned}
& \delta E_{x}=\sum_{k} \delta E(\mathbf{k}) \frac{k_{\perp}}{k} g_{k}(\mathbf{r}, t), \\
& \delta E_{y}=\sum_{k} \delta E(\mathbf{k})\left(\frac{k_{y} k_{x}}{k_{\perp} k} g_{k}(\mathbf{r}, t)+\frac{k_{z}}{k_{\perp}} h_{\boldsymbol{k}}(\mathbf{r}, t)\right), \\
& \delta E_{z}=\sum_{k} \delta E(\mathbf{k})\left(\frac{-k_{z} k_{x}}{k_{\perp} k} g_{k}(\mathbf{r}, t)-\frac{k_{y}}{k_{\perp}} h_{k}(\mathbf{r}, t)\right) .
\end{aligned}
$$

Here, the wave harmonics are $\mathrm{g}_{\mathbf{k}}=\cos \left(\mathbf{k r}+\varphi_{\mathbf{k}}^{1}-t \omega_{\mathbf{k}}\right) ; \mathbf{h}_{\mathbf{k}}$ $=\sin \left(\mathbf{k r}+\varphi_{\mathbf{k}}^{2}-t \omega_{\mathbf{k}}\right) ; \mathrm{k}_{\perp}=\sqrt{\mathrm{k}_{\mathrm{z}}^{2}+\mathrm{k}_{\mathrm{y}}^{2}} ;$ and $\mathrm{k}=\sqrt{\mathrm{k}_{\mathrm{x}}^{2}+\mathrm{k}_{\mathrm{y}}^{2}+\mathrm{k}_{\mathrm{z}}^{2}}$. We chose the initial phases $\varphi_{\mathbf{k}}^{1}, \varphi_{\mathbf{k}}^{2}$-randomly with uniform distribution over the interval $[0,2 \pi]$. In the present simulations, five hundred harmonics were launched into the system. The frequencies $\omega_{\mathbf{k}}$ and amplitudes $\delta E(\mathbf{k})$ of the waves were taken from Cluster satellite data (recorded by C4) on July 20, 2013; $k$ are distributed in the range of $\frac{2 \pi}{L} \cdot[0.05,4]$.

The components of magnetic field $\delta B_{x}, \delta B_{y}, \delta B_{z}$ can be obtained from Maxwell equations

$$
\begin{aligned}
\nabla \times \delta \mathbf{B} & =\frac{1}{c} \frac{\partial \delta \mathbf{E}}{\partial t}, \\
\nabla \cdot \delta \mathbf{B} & =0 .
\end{aligned}
$$

We considered a multicomponent plasma consisting of electrons $\mathrm{e}^{-}$, protons $\mathrm{H}^{+}$, helium $\mathrm{He}^{+}$, and oxygen $\mathrm{O}^{+}$ions, which were traced toward the current sheet having the thickness equal to $L_{z}=2000 \mathrm{~km}$. The modeling region is a $3-\mathrm{D}$ box with the following sizes for scenarios
A, C, and D: $z \in\left[-L_{z}, L_{z}\right]\left(L_{z}=2 \times 10^{3} \mathrm{~km}\right) ; x \in\left[-L_{x}, L_{x}\right]$; and $y \in\left[-L_{y}, L_{y}\right]$, where $L_{x}=7.5 \times 10^{4} \mathrm{~km}$ and $L_{y}=2 \cdot L_{x}=15 \times 10^{4} \mathrm{~km}$. For scenario $\mathrm{B}$, the characteristic size of a single dipolarization front was chosen $a s \approx 0.5 \mathrm{R}_{\mathrm{E}}$ in the dawn-dusk direction [Nakamura et al., 2004]. We have launched $\mathrm{N}=5 \times 10^{5}$ particles into the region of modeling. All quantities are normalized to the corresponding proton characteristics. The initial velocity distribution function has been taken in the form of a shifted kappa distribution that is typical for the plasma sheet of the Earth's magnetotail

$$
\mathrm{f}(\mathbf{v})=\frac{n_{0} A_{\kappa_{\varepsilon}}}{2\left(\sqrt{\pi k} V_{\kappa_{\varepsilon}}\right)^{3}} \cdot \sum_{s=1,2} 1+\frac{\mathbf{v}_{\perp}^{2}+\left(\mathbf{v}_{\|}-(-1)^{s} \mathbf{v}_{\mathrm{D}}\right)^{2-\left(\kappa_{\varepsilon}+1\right)}}{\kappa_{\varepsilon} \cdot v_{\kappa_{\varepsilon}}^{2}} .
$$

Here, $n_{0}$ is the plasma density; $v_{\kappa_{\varepsilon}}=v_{T}$ is the thermal velocity; $\kappa_{\varepsilon}$ is the parameter of the kappa distribution that was chosen to be equal to 3 to make the distribution (7) in accordance with the real one; and parameter $v_{D}=800 \mathrm{~km} / \mathrm{s}$ is the plasma flow velocity, i.e., macroscopic characteristic of the initial distribution (7).

Profiles of the normal magnetic component $B_{z}$ corresponding to four mechanisms at different temporal scales are shown in Figs. 1(a)-1(d). Temporal profiles were taken from the analysis of the Cluster dipolarization event observed on July 20, 2013 [Grigorenko et al., 2018; Malykhin et al., 2018], namely, (A) a single large-scale dipolarization with the duration of $t=3 \mathrm{~min}$ and the value of total magnetic jump $\Delta B_{z}=25 \mathrm{nT}$; (B) single dipolarization front with a total duration of $t \approx 1 \mathrm{~min}$ and a magnetic jump of $\Delta B_{z}=10 \mathrm{nT}$; (C) several dipolarization fronts with the average duration of about $t_{D f s}$ $\approx 20-30 \mathrm{~s}$ and a total duration of $t_{d} \approx 10 \mathrm{~min}$; and (D) multiple dipolarization fronts (case C) observed at the time scale $t_{D F s}$ followed by high-frequency magnetic field fluctuations on the electron gyroperiod scale observed in the time interval $t_{\text {elf }}=6.3 \mathrm{~min}$ and taken into account in the model by Eq. (6). We calculated maximum particle energies $\mathrm{E}_{\max }$ and average energies $\overline{\mathrm{E}}$ gained during the realization of scenarios (A)-(D) of magnetic perturbations. Also, the coefficient $\lambda=\frac{\mathrm{E}_{\max }}{\overline{\mathrm{E}}_{0}}$ characterizing the relative gain of the energies during the process of acceleration is analyzed.

\section{SIMULATION RESULTS}

Figures 2(a) and 2(b) illustrate the characteristic temporal behavior of energies by four different particles: electron $\mathrm{e}^{-}$, proton $\mathrm{H}^{+}$, helium ion $\mathrm{He}^{+}$, and oxygen ion $\mathrm{O}^{+}$during 4 different scenarios of magnetotail current sheet perturbations (A)-(D).

Figure 2(a) demonstrates the typical changes of energies of four single particles in the current sheet with (A) large-scale magnetic dipolarization. We see that the character of the energy increase for electrons is different from that for ions. Thus, the electron energy monotonously grows by two orders of magnitude and they finally gain energies up to $\sim 50 \mathrm{keV}$, but the saturation does not occur. In contrast, the acceleration of protons, helium ions, and oxygen ions passes quickly from energies of 6,8 , and $12 \mathrm{keV}$ up to about 100,150 , and $200 \mathrm{keV}$, respectively, with the subsequent saturation in the interval of 60-100 s.

Figure 2(b) illustrates the gain of energy by particles in the current sheet during the realization of mechanism B, i.e., single magnetic dipolarization. We see that particle energies grow monotonically, increasing from initial energies of $1,6,8$, and $12 \mathrm{keV}$ for electron $\mathrm{e}^{-}$, proton $\mathrm{H}^{+}$, helium ion $\mathrm{He}^{+}$, and oxygen $\mathrm{O}^{+}$ions to $30,50,60$, and $80 \mathrm{keV}$, respectively. 

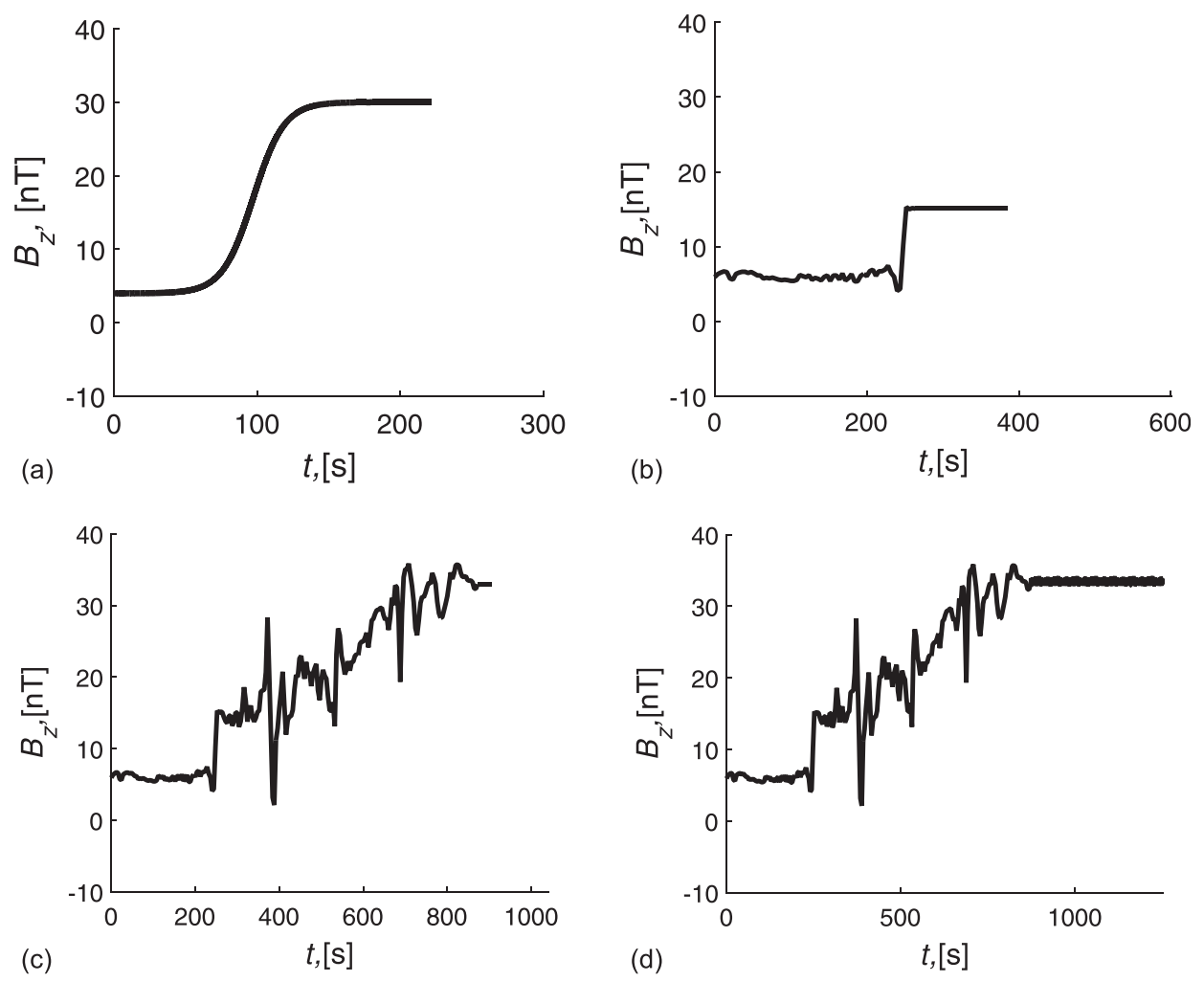

FIG. 1. Time profiles of dipolarization $\Delta \mathbf{B}$ corresponding to four scenarios of current sheet perturbations are shown: (a) single large-scale dipolarization with a total duration of $t=3 \mathrm{~min}$; (b) single dipolarization front with a total duration of $t \approx 1 \mathrm{~min}$; (c) dipolarization with a total duration of $t_{d} \approx 10 \mathrm{~min}$, including several dipolarization fronts with the average duration of about $t_{D f \mathrm{~s}} \approx 20-30 \mathrm{~s}$; and (d) multiple dipolarization fronts observed at the time scale $t_{D f s} \approx 20-30$ s followed by high-frequency electromagnetic field fluctuations observed during the interval $t_{\text {elf }}$ $=6.3 \mathrm{~min}$.
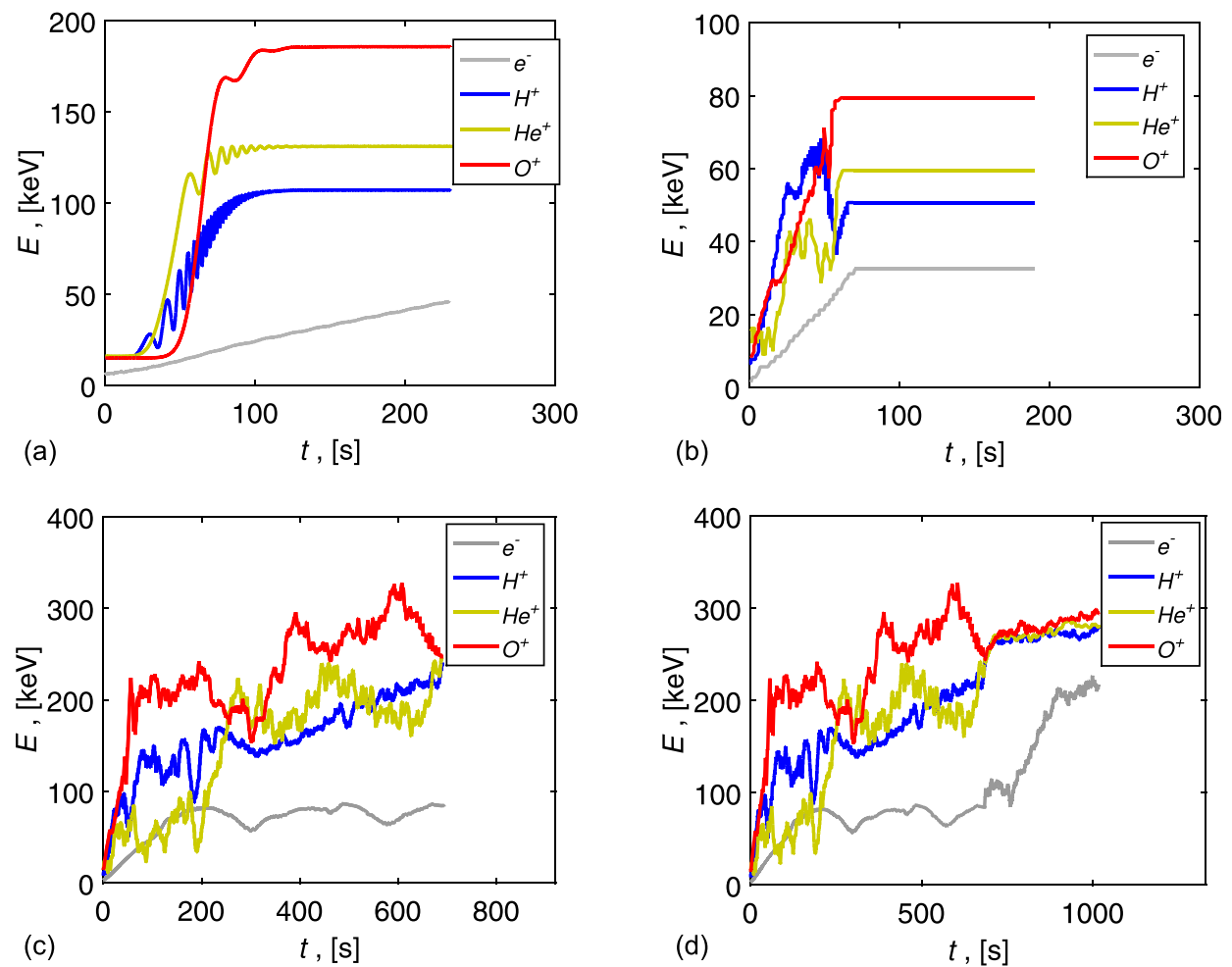

FIG. 2. Temporal changes of energies of the electron $\mathrm{e}^{-}$, proton $\mathrm{H}^{+}$, helium ion $\mathrm{He}^{+}$, and oxygen ion $\mathrm{O}^{+}$having the same initial positions and velocities during 4 different scenarios of magnetic perturbations: A [(a)], B [(b)], C [(c)], and D [(d)]. 
Figure 2(c) shows the dependences of the energy changes of the same 4 particles in the course of their interaction with the current sheet during the $\mathrm{C}$-scenario of the propagation of multiple dipolarization fronts. In this case, particle acceleration consists of sequences of energy increase and decrease in the process of interaction with dipolarization fronts. Electrons experience a strong acceleration from $1 \mathrm{keV}$ up to $\sim 100 \mathrm{keV}$. During the same time interval, protons, helium, and oxygen ions can be accelerated by dipolarization fronts from initial energies of 6,8 , and $12 \mathrm{keV}$ up to about 250,300 , and $350 \mathrm{keV}$, respectively.

During scenario D, as it is shown in Fig. 2(d), particle energies grow practically linearly till the time moment $t=680 \mathrm{~s}$, when highfrequency electromagnetic turbulence begins. After the termination of multiple dipolarizations, protons, helium, and oxygen ions do not experience any significant acceleration, while electrons continue to be effectively accelerated from $\sim 100 \mathrm{keV}$ up to $\sim 250 \mathrm{keV}$. This process has a character of resonance acceleration because the temporal characteristics of electromagnetic turbulence are about the electron gyroperiod which is much shorter than the gyroperiod of the other kinds of particles. The comparison of energy gain for scenarios A-D clearly demonstrates that different plasma particles may be effectively accelerated during different periods on the corresponding multiple temporal scales. While protons, helium, and oxygen ions are essentially affected by the induced electric field in the course of single or multiple dipolarization fronts, the most prominent electron acceleration occurs after termination of dipolarization fronts due to the beginning of electric and magnetic fluctuations.
To estimate quantitatively the efficiency of each from the four mechanisms (A)-(D), i.e., realization of a single large-scale dipolarization (case A), passage of a single fast front of dipolarization (case B), propagation of multiple dipolarization fronts (case $\mathrm{C}$ ), and passage of multiple dipolarization fronts accompanied by electromagnetic turbulence (case D), we present the energy spectra [see Figs. 3(a)-3(d)] of the electrons $\mathrm{e}^{-}$, protons $\mathrm{H}^{+}$, helium $\mathrm{He}^{+}$, and oxygen $\mathrm{O}^{+}$ions. The energy spectra in Fig. 3 confirm that all mechanisms contribute to the plasma acceleration; however, different types of particles can be accelerated on different temporal scales, comparable with their characteristic gyroperiods. First, one can see that all spectra have the decrease in small energies in comparison with the initial spectrum. This occurs due to particle acceleration processes when particles with low energies become more energized and "transfer" to the part of spectra with higher energies.

Figure 3(a) shows that acceleration by a total dipolarization mechanism (case A) can lead to the energy gain by one order of value or slightly more for all types of particles. As expected, there is no significant energization of all types of particles in case B in comparison with case C. It should be noted that an additional peak that can be seen in the spectrum of accelerated particles in Fig. 3(b) indicates a resonance acceleration effect, which was discussed earlier in the literature [Grigorenko et al., 2017; Malykhin et al., 2018]. This passage of a single dipolarization front can lead to the appearance of a population of strongly accelerated particles in the finite energy range, which results in a localized peak in the energy spectra. For protons, helium, and oxygen ions, the passage of multiple fronts of dipolarization (case C) having a duration of less than 1 min leads to the resonant interactions and

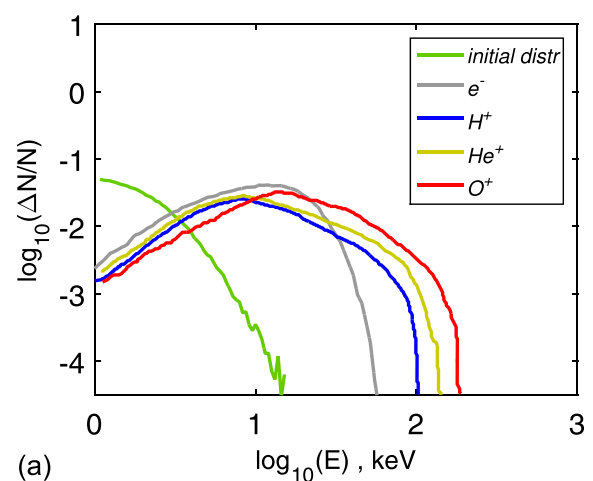

(a)

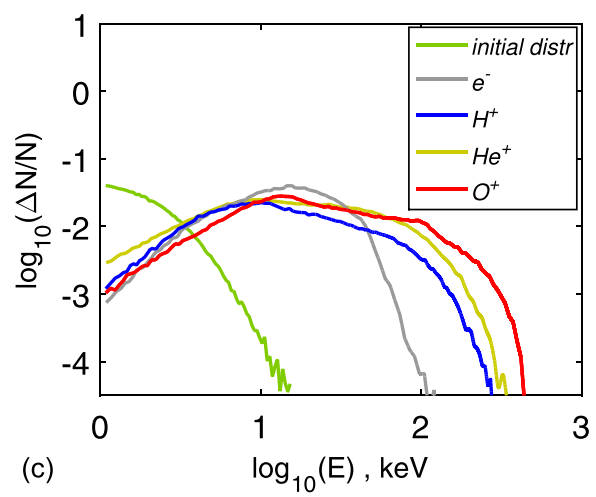

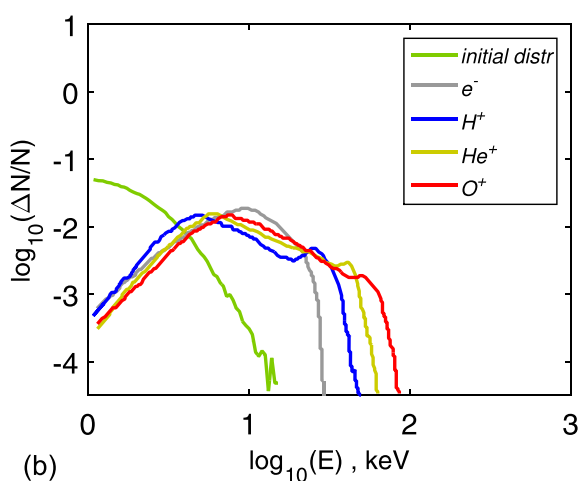

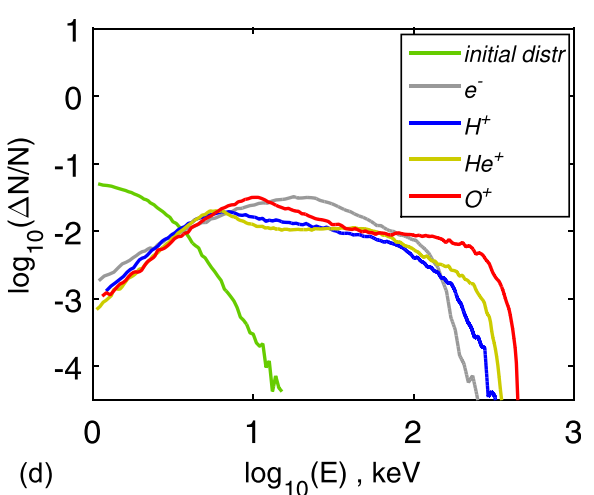

FIG. 3. Energy spectra of $\mathrm{e}^{-}, \mathrm{H}^{+}, \mathrm{He}^{+}$, and $\mathrm{O}^{+}$in the case of: (a) single largescale dipolarization; (b) single dipolarization front; (c) multiple dipolarization fronts; (d) multiple dipolarization fronts followed by electric and magnetic fluctuations. 
TABLE I. Energetic characteristics of plasma particles before and after acceleration by (from top to bottom): (A) a large-scale dipolarization, (B) single dipolarization front, (C) multiple dipolarization fronts, and (D) like case B but followed by electric and magnetic fluctuations. The right column shows the coefficient $\lambda=\mathrm{E}_{\max } / \overline{\mathrm{E}}_{0}$ which characterizes the relative gain of the energies.

\begin{tabular}{|c|c|c|c|c|c|c|c|}
\hline Acceleration process & Particles & $\overline{\mathrm{E}}_{0}[\mathrm{keV}]$ & $\overline{\mathrm{E}}[\mathrm{keV}]$ & $\mathrm{E}_{\max }[\mathrm{keV}]$ & $\Delta \mathrm{E}=\mathrm{E}_{\mathrm{fin}}-\mathrm{E}_{\mathrm{ini}},[\mathrm{keV}]$ & $\lambda$ & $\gamma$ \\
\hline \multirow[t]{4}{*}{ (A) } & $\mathrm{e}^{-}$ & 1 & 11 & 60 & 10 & 60 & 10 \\
\hline & $\mathrm{H}^{+}$ & 6 & 7 & 100 & 1 & 16 & 0.16 \\
\hline & $\mathrm{He}^{+}$ & 8 & 9 & 150 & 1 & 18 & 0.12 \\
\hline & $\mathrm{O}^{+}$ & 12 & 13 & 200 & 1 & 16 & 0.08 \\
\hline \multirow[t]{4}{*}{ (B) } & $\mathrm{e}^{-}$ & 1 & 10 & 30 & 9 & 30 & 9 \\
\hline & $\mathrm{H}^{+}$ & 6 & 8 & 50 & 2 & 8 & 0.33 \\
\hline & $\mathrm{He}^{+}$ & 8 & 10 & 65 & 2 & 8 & 0.25 \\
\hline & $\mathrm{O}^{+}$ & 12 & 15 & 80 & 3 & 6 & 0.25 \\
\hline \multirow[t]{4}{*}{ (C) } & $\mathrm{e}^{-}$ & 1 & 12 & 150 & 11 & 150 & 11 \\
\hline & $\mathrm{H}^{+}$ & 6 & 8 & 300 & 2 & 50 & 0.33 \\
\hline & $\mathrm{He}^{+}$ & 8 & 9 & 350 & 1 & 43 & 0.12 \\
\hline & $\mathrm{O}^{+}$ & 12 & 15 & 450 & 3 & 37 & 0.25 \\
\hline \multirow[t]{4}{*}{ (D) } & $\mathrm{e}^{-}$ & 1 & 20 & 250 & 19 & 250 & 19 \\
\hline & $\mathrm{H}^{+}$ & 6 & 8 & 320 & 2 & 53 & 0.33 \\
\hline & $\mathrm{He}^{+}$ & 8 & 9 & 360 & 1 & 45 & 0.12 \\
\hline & $\mathrm{O}^{+}$ & 12 & 15 & 500 & 3 & 41 & 0.25 \\
\hline
\end{tabular}

energy transfer from the field to the particles [Figs. 2(c) and 3(c)]. Here, electrons are less affected because of their small gyroperiods. In contrast, high-frequency electric and magnetic fluctuations following the passage of dipolarization fronts (case D) can effectively lead to the resonant acceleration of electrons [Fig. 3(d)] with gain of energies about two orders of magnitude. In other words, combination of DF passage and electric and magnetic fluctuations appears as the most effective mechanism of particle acceleration.

Parameters of accelerated particles are shown in Table I, where one can see energetic characteristics of electrons, protons, helium, and oxygen ions (initial average energy $\overline{\mathrm{E}}_{0}$; final average energy $\overline{\mathrm{E}}$, maximum energy $E_{\text {max }}$, the relative average energy gain $\gamma=\Delta \mathrm{E} / \overline{\mathrm{E}}_{0}$, and the coefficient $\lambda=\mathrm{E}_{\max } / \overline{\mathrm{E}}_{0}$ characterizing the ratio of the maximum energy to the initial one) before and after their interaction with magnetotail perturbation. Magnetotail values of the initial particle energies $\overline{\mathrm{E}}$ which are typical were chosen corresponding to Cluster data on July 20, 2013 (from 01:33:08 to 01:48:11 UT) and equal to $\overline{\mathrm{E}}=1,6,8$, and $12 \mathrm{keV}$ for $\mathrm{e}^{-}, \mathrm{H}^{+}, \mathrm{He}^{+}$, and $\mathrm{O}^{+}$, respectively.

Table I summarizes energetic characteristics obtained for electrons, protons, helium, and oxygen ions, viz., initial average energy $\overline{\mathrm{E}}_{0}$, final average energy $\overline{\mathrm{E}}$, maximum energy $\mathrm{E}_{\max }$, relative average energy gain $\gamma=\Delta \mathrm{E} / \overline{\mathrm{E}}_{0}$, and coefficient $\lambda=\mathrm{E}_{\max } / \overline{\mathrm{E}}_{0}$ which characterizes the ratio of the maximum energy to the initial one. Typical values of the average thermal energies $\overline{\mathrm{E}}$ of $\mathrm{e}^{-}, \mathrm{H}^{+}, \mathrm{He}^{+}$, and $\mathrm{O}^{+}$were chosen to be consistent with Cluster data on July 20, 2013 (from 01:33:08 to 01:48:11 UT) and equal correspondingly to $\overline{\mathrm{E}}=1,6,8$, and $12 \mathrm{keV}$.

Using Table I, let us compare the average and maximum energy gain relatively initial energies for different kinds of particles after their interaction with the perturbed current sheet in scenarios A-D. Electrons with the average initial energy of $1 \mathrm{keV}$ can be accelerated to energies of $60-250 \mathrm{keV}$, which is one or two orders larger than the initial state. Coefficient $\gamma=\Delta \mathrm{E} / \overline{\mathrm{E}}_{0}$ is equal to 10,9 , and 11 in the corresponding scenarios $\mathrm{A}, \mathrm{B}$, and $\mathrm{C}$. In case $\mathrm{D}$, their acceleration coefficient $\gamma$ reached the value of 19. The comparison of Figs. 1(c) and 1(d) shows the substantial contribution of the mechanism of particle acceleration by the electromagnetic turbulent fluctuations with the characteristic temporal scale about the electron gyroperiods. The coefficient of maximum acceleration $\lambda$ demonstrated the same dependence: its values are follows: $60,30,150$, and 250 (scenarios A, B, C, and D). Also, one can see that multiple repetitions of dipolarizations (B) are more effective than a single one. Because electrons are almost magnetized in the system, this comparison also shows that betatron acceleration can be a general mechanism at the initial period of time $0<$ $\mathrm{t}<680 \mathrm{~s}$ before the beginning of turbulence. Comparison of Figs. 1(d) and 1 (c) confirms this conclusion: we see that electrons are much effectively accelerated by the induced electric field of multiple polarizations at this period of time.

As for the relative gain of energy of protons and heavy ions, in general, it is much less in comparison with the electron ones since these particles are more massive. Values of the coefficient $\gamma=0.16$, $0.33,0.33,0.33$ for protons (scenarios $\mathrm{A}-\mathrm{D}$ ) show that fast dipolarization events (including scenario $\mathrm{B}$ of a single perturbation) in the magnetotail are much more effective than a single quite slow dipolarization with the duration of $3 \mathrm{~min}$. The reason for that is the coincidence of the temporal scales of magnetic dipolarizations and proton gyroperiods $(\sim 1 \mathrm{~min})$ when the resonance transfer of energy to particles is maximum. Relative maximum energies of proton acceleration in cases $\mathrm{A}, \mathrm{C}$, and $\mathrm{D}$ are equal to $\gamma=16,50$, and 53 (absolute values of energies are 100,300 , and $320 \mathrm{keV}$ ), respectively, which shows the efficiency of mechanisms $\mathrm{C}$ and $\mathrm{D}$ of multiple dipolarizations. We also see that there is almost no difference between acceleration parameters in cases $\mathrm{C}$ and $\mathrm{D}$; the reason for this is the absence of resonance 
between proton gyroperiods and much faster electromagnetic turbulence.

Almost the same behavior is characteristic of the acceleration of oxygen ions; for them, the values of coefficients $\gamma$ and $\lambda$ are smallest (oxygen ions are the heaviest) and equal, correspondingly $\gamma=0.08$, $0.25,0.25,0.25$ and $\lambda=16,6,37,41$ (scenarios A, B, C, and D). Their final energies are several times greater than the initial ones, and practically, there is no difference between mechanisms C and D. Gain of energy of helium ions is several times larger in C and D mechanisms in comparison with $\mathrm{A}$, and as for protons, we do not see the difference between acceleration in C and D scenarios. Summing up the results of this comparison, we can say that acceleration of different kinds of particles in the multicomponent plasma of the Earth's magnetotail during substorm dipolarizations has a resonance character. In the mixture of particle populations, maximally accelerated populations have gyroperiods comparable to the temporal scale of magnetic disturbances.

There exists some difference in the character of acceleration in case B from other scenarios A, C, and D. It can be explained from the point of view of the spatial configuration of region where particles interact with a magnetic perturbation (interactive region). As one can see above in numerical calculations, the size of the box for three scenarios $\mathrm{A}, \mathrm{C}$, and $\mathrm{D}$ (about $20 \mathrm{R}_{\mathrm{E}}$ ) was much higher than that in case $\mathrm{B}$ $\left(\sim 0.5 \mathrm{R}_{\mathrm{E}}\right)$. In the first case, we considered the full cross-tail size of the magnetosphere along the $\mathrm{Y}$ coordinate. In the last case, scaling was chosen according to the available experimental observations of passage of single dipolarization front in the magnetotail current sheet (see above). Thus, the difference in modeling results in scenario B in comparison with $\mathrm{A}, \mathrm{C}$, and $\mathrm{D}$ can be explained from the point of view of the spatial size of the interactive region rather than the abovementioned temporal resonance. Coefficients of relative maximum energies $\lambda$ after a single dipolarization (B) for both electrons, protons, helium, and oxygen were $30,8,8$, and 6 versus $C$ and $D$ scenarios, respectively, where this coefficient was about 5 times larger. It is known that energized particles tend to leave the interactive region when their Larmor radii become about the scale of a magnetic inhomogeneity. This spatial restriction leads to the limitation on the maximum energies of all particles. One can see in Figs. 2(a)-2(d) that all energy spectra of different kinds of particles have steep falling profiles on higher energies. This effect is due to the particle escape from the interactive region.

\section{DISCUSSION AND CONCLUSIONS}

In this work, we focused on the effectiveness of particle energization during dipolarization events that can have a form of a total dipolarization or can have a complex multiscale character due to the set of piled-up dipolarization fronts, followed sometimes by consequent excitation of electric and magnetic fluctuations. Generally, acceleration processes depend, on the one hand, on the spatial and temporal properties of the system and, on the other hand, on particle charges, masses, and their initial energy distributions. Thus, the acceleration processes have complex and nonlinear multiscale character. Scenarios A, C, and D are different from scenario B by their spatial characteristics. In scenario $\mathrm{B}$, the $\mathrm{Y}$-size of the acceleration region was $0.5 \mathrm{RE}$, whereas for the remaining scenarios, the corresponding lengths were considered equal to $7.5 \times 10^{4} \mathrm{~km}$. The B scenario of particle acceleration seems to be the least effective for particles of all masses. This follows from a comparison of particle energy maxima in Table I, where they are much smaller in comparison with other scenarios. Therefore, the size of the acceleration region in scenario B is so small that particles leave it without significant acceleration.

At the same time, the comparison of acceleration processes in scenarios A, C, and D, where the sizes of the system are the same (and much larger than in the B case), shows that the acceleration of particles strongly depends on the proximity of characteristic scales of particle gyration and temporal changes of magnetic and electric fields in the system. Our investigations have shown that the singleand multiscale changes of magnetic fields lead to the generation of induced electric fields that interact with plasma particles and accelerate them in a resonant manner. Thus, the closer the time scale of the field variation to the particle gyroperiod, the more effective is the transfer of energy from fields to particles. Four main mechanisms occurring at different time scales considered here are as follows: (A) general magnetic field dipolarization with a relatively slow duration of about three minutes, (B) single dipolarization front observed at smaller time scales less than one minute and the limited spatial scale about $0.5 \mathrm{R}_{\mathrm{E}}$, (C) passage of multiple dipolarization fronts with the full time scale about four minutes, and (D) the case of multiple dipolarizations fronts followed by high-frequency fluctuations of electric and magnetic fields near electron cyclotron frequencies. Generally, it is shown that

(1) Oxygen ions are more efficiently accelerated by the induced electric field during the slower total dipolarization event (mechanism A) but are less sensitive than protons and helium ions to pile-up many dipolarization fronts on the shorter time scales less than 1 min (B, C, D).

(2) Protons and helium ions can be significantly accelerated by mechanisms (A) and (C), but the maximum net energy gain was found during last mechanism (C).

(3) Electrons are less sensitive than ions to mechanisms (A) and (C) but are efficiently accelerated by fast electric field fluctuations (scenario D).Thus, electrons can most effectively be accelerated after the end of large-scale multiple dipolarizations when electromagnetic turbulence with close temporal parameters begins.

(4) Mechanism (B) is the least efficient acceleration mechanism for protons, helium, and oxygen ions, the corresponding coefficients of maximum energy gains of which are 8,8 , and 6 , respectively.

(5) In the case of a spatially limited size of the system (scenario B), energy spectra can have a specific local maximum in the central part due to resonance acceleration of that population with gyroradii comparable with the size of the system. The small size of a single dipolarization front indicates that this mechanism is the least effective in plasma acceleration in comparison with more large-scale dipolarization mechanisms $\mathrm{A}, \mathrm{C}$, and $\mathrm{D}$, where the sizes of the acceleration region are much larger.

(6) Generally, the shapes of particle energy spectra can be interpreted by the contribution of different mechanisms in the total acceleration process, having temporal and scale resonance nature. After particle interaction with magnetic perturbations, the initial Maxwellian spectra are transformed to power-law profiles with three clearly seen slopes from smaller to higher energies: positive, almost plateau, and negative one. At small energies, all spectra have a positive slope, and these profiles grow linearly to higher energies because most of the low-energy particles are accelerated and cannot contribute to this diapason of energies. Plateau of energy spectra is due to the influence of different scenarios of acceleration A-D. At the highest values of energies, one can see the sharp decrease of the spectra profile for almost all kinds of particles because of the spatial 
limitation of a system in the Y direction, where particles can be trapped and multiply accelerated before escaping in the loss cone.

\section{ACKNOWLEDGMENTS}

The work by E.I.P., E.E.G., V.Yu.P., E.A.K., and P.V.D. was done in the frame of Volkswagen Foundation Grant No. Az90 312. H.V.M. acknowledges RFBR Grant Nos. 19-02-00957 and 17-02-01328.

\section{REFERENCES}

Angelopoulos, V., Runov, A., Zhou, X. Z., Turner, D. L., Kiehas, S. A., Li, S.-S., and Shinohara, I., "Electromagnetic energy conversion at reconnection fronts," Science 341, 1478-1482 (2013).

Apatenkov, S. V., Sergeev, V. A., Kubyshkina, M. V., Nakamura, R., Baumjohann, W., Runov, A., Alexeev, I., Fazakerley, A., Frey, H., Muhlbachler, S., Daly, P. W., Sauvaud, J. A., Ganushkina, N., Pulkkinen, T., Reeves, G. D., and Khotyaintsev, Y., "Multi-spacecraft observation of plasma dipolarization/ injection in the inner magnetosphere," Ann. Geophys. 25, 801-814 (2007).

Artemyev, A. V., Lutsenko, V. N., and Petrukovich, A. A., "Ion resonance acceleration by dipolarization fronts: Analytic theory and spacecraft observation," Ann. Geophys. 30, 317-324 (2012).

Ashour-Abdalla, M., Lapenta, G., Walker, R. J., El-Alaoui, M., and Liang, H., "Multiscale study of electron energization during unsteady reconnection events," J. Geophys. Res. 120, 4784-4799, https://doi.org/10.1002/2014JA020316 (2015).

Baker, D. N., Fritz, T. A., McPherron, D., Fairfield, H., Kamide, Y., and Baumjohann, W., J. Geophys. Res. 90, 1205-1216, https://doi.org/10.1029/ JA090iA02p01205 (1985).

Baker, D. N. et al., "Timing of magnetic reconnection initiation during a global magnetospheric substorm onset," Geophys. Res. Lett. 29(24), 2190, https:// doi.org/10.1029/2002GL015539 (2002).

Birn, J., Thomsen, M. F., and Hesse, M., "Electron acceleration in the dynamic magnetotail: Test particle orbits in three-dimensional magnetohydrodynamic simulation fields," J. Plasma Phys. 11, 1825-1833 (2004).

Birn, J., Artemyev, A. V., Baker, D. N., Echim, M., Hoshino, M., and Zelenyi, L. M., "Particle acceleration in the magnetotail and aurora," Space Sci. Rev. 173, 49-102 (2012).

Birn, J., Hesse, M., Nakamura, R., and Zaharia, S., "Particle acceleration in dipolarization events," J. Geophys. Res. Space Phys. 118, 1960-1971, https://doi.org/ 10.1002/jgra.50132 (2013).

Borg, A. L., Øieroset, M., Phan, T. D., Mozer, F. S., Pedersen, A., Mouikis, C., McFadden, J. P., Twitty, C., Balogh, A., and Réme, H., "Cluster encounter of a magnetic reconnection diffusion region in the near-Earth magnetotail on September 19, 2003,” J. Geophys. Res. 32, L19105, https://doi.org/10.1029/ 2005GL023794 (2005).

Catapano, F., Zimbardo, G., Perri, S., Greco, A., and Artemyev, A. V., "Proton and heavy ion acceleration by stochastic fluctuations in the Earth's magnetotail," Ann. Geophys. 34, 917-926 (2016).

Delcourt, D. C., Pedersen, A., and Sauvaud, J.-A., "Dynamics of single-particle orbits during substorm expansion phase,” J. Geophys. Res. 95, 20853-20865, https://doi.org/10.1029/JA095iA12p20853 (1990).

Delcourt, D. C., "Particle acceleration by inductive electric fields in the inner magnetosphere," J. Atmos. Sol. Terr. Phys. 64, 551-559 (2002).

Drake, J. F., Swisdak, M., Phan, T. D., Cassak, P. A., Shay, M. A., Lepri, S. T., Lin, R. P., Quataert, E., and Zurbuchen, T. H., "Ion heating resulting from pickup in magnetic reconnection exhausts," J. Geophys. Res. 114, A05111, https:// doi.org/10.1029/2008JA013701 (2009).

El-Alaoui, M., Richard, R. L., Ashour-Abdalla, M., Goldstein, M. L., and Walker, R. J., "Dipolarization and turbulence in the plasma sheet during a substorm: THEMIS observations and global MHD simulations," J. Geophys. Res. Space Phys. 118, 7752-7761, https://doi.org/10.1002/2013JA019322 (2013).

Fu, H. S., Khotyaintsev, Y. V., Andre, M., and Vaivads, A., "Fermi and betatron acceleration of suprathermal electrons behind dipolarization fronts," Geophys. Res. Lett. 38, L16104, https://doi.org/10.1029/2011GL048528 (2011).

Galeev, A. A., "The mechanism of magnetosphere substorms," Sov. Phys. Usp. 22, 196-197 (1979).
Greco, A., Artemyev, A., and Zimbardo, G., "Heavy ion acceleration at dipolarization fronts in planetary magnetotails," Geophys. Res. Lett. 42, 8280-8287, https://doi.org/10.1002/2015GL066167 (2015).

Greco, A., Perri, S., Zimbardo, G., and Zelenyi, L. M., "Particle acceleration by stochastic fluctuations and dawn-dusk electric field in the Earth's magnetotail," Adv. Space Res. 44, 528-533 (2009).

Grigorenko, E. E., Hoshino, M., Hirai, M., Mukai, T., and Zelenyi, L. M., "Geography of ion acceleration in the magnetotail: X-line versus current sheet effects," J. Geophys. Res. 114, A03203, https://doi.org/10.1029/2008JA013811 (2009).

Grigorenko, E. E., Zelenyi, L. M., Dolgonosov, M. S., Artemiev, A. V., Owen, C. J., Sauvaud, J. A., Hoshino, M., and Hirai, M., "Non-adiabatic ion acceleration in the earth magnetotail and its various manifestations in the plasma sheet boundary layer," Space Sci. Rev. 164, 133-181 (2011).

Grigorenko, E. E., Malykhin, A. Y., Kronberg, E. A., Malova, K. V., and Daly, P. W., "Acceleration of ions to suprathermal energies by turbulence in the plasmoid-like magnetic structures," J. Geophys. Res. Space Phys. 120, 6541-6558, https://doi.org/10.1002/2015JA021314 (2015).

Grigorenko, E. E., Kronberg, E. A., Daly, P. W., Ganushkina, N. Y., Lavraud, B., Sauvaud, J.-A., and Zelenyi, L. M., "Origin of low proton-to-electron temperature ratio in the Earth's plasma sheet," J. Geophys. Res. Space Phys. 121, 9985-10,004, https://doi.org/10.1002/2016JA022874 (2016).

Grigorenko, E. E., Kronberg, E. A., and Daly, P. W., "Heating and acceleration of charged particles during magnetic dipolarizations," Cosmic Res. 55, 57-66 (2017).

Grigorenko, E. E., Dubyagin, S., Malykhin, A. Y., Khotyaintsev, Y. V., Kronberg, E. A., Lavraud, B., and Ganushkina, N. Y., "Intense current structures observed at electron kinetic scales in the near-Earth magnetotail during dipolarization and substorm current wedge formation," Geophys. Res. Lett. 45(2), 602-611, https://doi.org/10.1002/2017GL076303 (2018).

Harris, E. G., "On a plasma sheath separating regions of oppositely directed magnetic fields," II Nuovo Chimento 23, 115-119 (1962).

Heyn, M. F. and Semenov, V. S., "Rapid reconnection in compressible plasma," J. Plasma Phys. 3, 2725-2741 (1996).

Hoshino, M., Mukai, T., Yamamoto, T., and Kokubun, S., "Ion dynamics in magnetic reconnection: Comparison between numerical simulation and Geotail observations," J. Geophys. Res. 103, 4509-4530, https://doi.org/10.1029/ 97JA01785 (1998).

Hoshino, M., Mukai, T., Nishida, A., Kokubun, S., and Yamamoto, T., "Nongyrotropic ions as evidence for an X-type neutral region," Adv. Space Res. 26, 425-430 (2000).

Hoshino, M., "Electron surfing acceleration in magnetic reconnection," J. Geophys. Res.: Space Phys. 110, A10215, https://doi.org/10.1029/ 2005JA011229 (2005).

Ipavich, F. M., Galvin, A. B., Gloeckler, G., Hovestadt, D., Klecker, B., and Scholer, M., "Energetic (greater than $100 \mathrm{keV}) \mathrm{O}(+)$ ions in the plasma sheet," Geophys. Res. Lett. 11, 504-507, https://doi.org/10.1029/GL011i005p00504 (1984).

Kiehas, S. A., Angelopoulos, V., Runov, A., and Li, S.-S., "On the azimuthal size of flux ropes near lunar orbit,” J. Geophys. Res. Space Phys. 118, 4415-4424, https://doi.org/10.1002/jgra.50425 (2013).

Kronberg, E. A., Grigorenko, E. E., Turner, D. L., Daly, P. W., Khotyaintsev, Y., and Kozak, L., "Comparing and contrasting dispersionless injections at geosynchronous orbit during a substorm event," J. Geophys. Res. 122, 3055-3072, https://doi.org/10.1002/2016JA023551 (2017).

Lembege, B. and Pellat, R., "Stability of a thick two-dimensional quasineutral sheet," Phys. Fluids 25, 1995-2004 (1982).

Liang, H., Lapenta, G., Walker, R. J., Schriver, D., El-Alaoui, M., and Berchem, J., "Oxygen acceleration in magnetotail reconnection," J. Geophys. Res. 122, 618-639, https://doi.org/10.1002/2016JA023060 (2017).

Longcope, D. W. and Priest, E. R., "Fast magnetosonic waves launched by transient, current sheet reconnection," J. Plasma Phys 14, 122905 (2007).

Lui, A. T. Y., Williams, D. J., Christon, S. P., McEntire, R. W., Angelopoulos, V., Jaquuey, C., Yamamoto, T., and Kokubun, S., “A preliminary assessment of energetic ion species in flux ropes/plasmoids in the distant tail," Geophys. Res. Lett. 21, 3019-3022, https://doi.org/10.1029/94GL01283 (1994).

Lui, A. T. Y., "Evidence for two types of dipolarization in the earth's magnetotail,” J. Atmos. Sol. Terr. Phys. 115, 17-24 (2014). 
Malykhin, A. Y., Grigorenko, E. E., Kronberg, E. A., Koleva, R., Ganushkina, N. Y., Kozak, L., and Daly, P. W., "Contrasting dynamics of electrons and protons in the near-Earth plasma sheet during dipolarizations," Ann. Geophys. 36, 741-760 (2018).

Nakamura, R., Baumjohann, W., Klecker, B., Bogdanova, Y., Balogh, A., Reme, H., Bosqued, J. M., Dandouras, I., Sauvaud, J. A., Glassmeier, K. H., Kistler, L., Mouikis, C., Zhang, T. L., Eichelberger, H., and Runov, A., "Motion of the dipolarization front during a flow burst event observed by Cluster," Geophys. Res. Lett. 29, 3-1, https://doi.org/10.1029/2002GL015763 (2002).

Nakamura, R. et al., "Spatial scale of high speed flows in the plasma sheet observed by Cluster," Geophys. Res. Lett. 31, L09804, https://doi.org/10.1029/ 2004GL019558 (2004).

Nakamura, R., Baumjohann, W., Asano, Y., Runov, A., Balogh, A., Owen, C. J., Fazakerley, A. N., Fujimoto, M., Klecker, B., and Réme, H., "Dynamics of thin current sheets associated with magnetotail reconnection," J. Geophys. Res. 111, A11206, https://doi.org/10.1029/2006JA011706 (2006).

Nakamura, R., Baumjohann, W., Fujimoto, M., Asano, Y., Runov, A., Owen, C. J., Fazakerley, A. N., Klecker, B., ReMe, H., Lucek, E. A., Andre, M., and Khotyaintsev, Y., "Cluster observations of an ion-scale current sheet in the magnetotail under the presence of a guide field," J. Geophys. Res. Space Phys. 113, A07S16, https://doi.org/10.1029/2007JA012760 (2008).

Nosé, M., Lui, A. T. Y., Ohtani, S., Mauk, B. H., McEntire, R. W., Williams, D. J., Mukai, T., and Yumoto, K., "Acceleration of oxygen ions of ionospheric origin in the near-Earth magnetotail during substorms," J. Geophys. Res. 105, 7669-7677, https://doi.org/10.1029/1999JA000318 (2000).

Ono, Y., Nose, M., Christon, S. P., and Lui, A. T. Y., "The role of magnetic field fluctuations in nonadiabatic acceleration of ions during dipolarization," J Geophys. Res. 114, A05209, https://doi.org/10.1029/2008JA013918 (2009).

Perri, S., Lepreti, F., Carbone, V., and Vulpiani, A., "Dynamical properties of test particles in stochastic electromagnetic fields," Commun. Nonlinear Sci. Numer. Simul. 14, 2347-2352 (2009).

Petkaki, P., Freeman, M. P., and Walsh, A. P., "Cluster observations of broadband electromagnetic waves in and around a reconnection region in the Earth's magnetotail current sheet," Geophys. Res. Lett. 33, L16105, https://doi.org/10.1029/ 2006GL027066 (2006).

Retino, A., Nakamura, R., Vaivads, A., Khotyaintsev, Y., Hayakawa, T., Tanaka, K., Kasahara, S., Fujimoto, M., Shinohara, I., Eastwood, J. P., Andre, M., Baumjohann, W., Daly, P. W., Kronberg, E. A., and Cornilleau-Wehrlin, N., "Cluster observations of energetic electrons and electromagnetic fields within a reconnecting thin current sheet in the Earth's magnetotail," J. Geophys. Res. Space Phys. 113, A12215, https://doi.org/10.1029/2008JA013511 (2008).

Richardson, I. G., Cowley, S. W. H., Hones, E. W., Jr., and Bame, S. J., "Plasmoidassociated energetic ion bursts in the deep geomagnetic tail: Properties of plasmoid and the postplasmoid plasma sheet," J. Geophys. Res. 92, 9997-10013, https://doi.org/10.1029/JA092iA09p09997 (1987).

Runov, A. et al., "Current sheet structure near magnetic X-line observed by Cluster," Geophys. Res. Lett. 30(11), 1579, https://doi.org/10.1029/2002GL016730 (2003).

Runov, A., Angelopoulos, V., Sergeev, V. A., Glassmeier, K. H., Auster, U., McFadden, J., Larson, D., and Mann, I., "Global properties of magnetotail current sheet flapping: THEMIS perspectives," Ann. Geophys. 27, 319-328 (2009).

Runov, A., Angelopoulos, V., Sitnov, M., Sergeev, V. A., Nakamura, R., Nishimura, Y., Frey, H. U., McFadden, J. P., Larson, D., Bonnell, J., Glassmeier, K. H., Auster, U., Connors, M., Russell, C. T., and Singer, H. J., "Dipolarization fronts in the magnetotail plasma sheet," Planet. Space Sci. 59, 517-525 (2011).

Runov, A., Angelopoulos, V., Zhou, X. Z., Zhang, X. J., Li, S., Plaschke, F., and Bonnell, J. A., "THEMIS multicase study of dipolarization fronts in the magnetotail plasma sheet," J. Geophys. Res. 116, A05216, https://doi.org/10.1029/ 2010JA016316 (2011).

Runov, A., Sergeev, V. A., Angelopoulos, V., Glassmeier, K. H., and Singer, H. J., J. Geophys. Res. Space Phys. 119, 1643-1657, https://doi.org/10.1002/ 2013JA019384 (2014).
Semenov, V. S., Penz, T., Ivanova, V. V., Sergeev, V. A., Biernat, H. K., Nakamura, R., Heyn, M. F., Kubyshkin, I. V., and Ivanov, I. B., "Reconstruction of the reconnection rate from cluster measurements: First results," J. Geophys. Res. Space Phys. 110, A11217, https://doi.org/10.1029/2005JA011181 (2005).

Sergeev, V. A., Elphic, R. C., Mozer, F. S., Saint-Marc, A., and Sauvaud, J. A., "A two satellite study of nightside flux transfer events in the plasma sheet," Planet. Space Sci. 40, 1551-1572 (1992).

Sergeev, V., Angelopoulos, V., Apatenkov, S., Bonnell, J., Ergun, R., Nakamura, R., McFadden, J., Larson, D., and Runov, A., "Kinetic structure of the sharp injection/dipolarization front in the flow-braking region," Geophys. Res. Lett. 36, L21105, https://doi.org/10.1029/2009GL040658 (2009).

Sitnov, M. I., Swisdak, M., and Divin, A. V., "Dipolarization fronts as a signature of transient reconnection in the magnetotail," J. Geophys. Res. 114, A04202, https://doi.org/10.1029/2008JA013980 (2009).

Sitnov, M. I. and Swisdak, M., "Onset of collisionless magnetic reconnection in two-dimensional current sheets and formation of dipolarization fronts," J Geophys. Res. 116, A12216, https://doi.org/10.1029/2011JA016920 (2011).

Sharma, A. S., Nakamura, R., Runov, A., Grigorenko, E. E., Hasegawa, H., Hoshino, M., Louarn, P., Owen, C. J., Petrukovich, A., Sauvaud, J.-A., Semenov, V. S., Sergeev, V. A., Slavin, J. A., Sonnerup, B. U. O., Zelenyi, L. M., Fruit, G., Haaland, S., Malova, H., and Snekvik, K., "Transient and localized processes in the magnetotail," Rev. Ann. Geophys. 26, 1-51 (2008).

Slavin, J. A., Owen, C. J., Dunlop, M. W., Boralv, E., Moldwin, M. B., Sibeck, D. G., Tanskanen, E., Goldstein, M. L., Fazakerley, A., Balogh, A., Lucek, E., Richter, I., Reme, H., and Bosqued, J. M., "Cluster four spacecraft measurements of small traveling compression regions in the near-tail," Geophys. Res. Lett. 30.23, 2208-2211 (2003).

Ukhorskiy, A. Y., Sitnov, M. I., Merkin, V. G., Gkioulidou, M., and Mitchell, D. G., "Ion acceleration at dipolarization fronts in the inner magnetosphere," J. Geophys. Res. Space Phys. 122, 3040-3054, https://doi.org/ 10.1002/2016JA023304 (2017).

Veltri, P., Zimbardo, G., Taktakishvili, A. L., and Zelenyi, L. M., "Effect of magnetic turbulence on the ion dynamics in the distant magnetotail," J. Geophys. Res. 103, 14897-14916, https://doi.org/10.1029/98JA00211 (1998).

Wygant, J. R. et al., "Cluster observations of an intense normal component of the electric field at a thin reconnecting current sheet in the tail and its role in the shock-like acceleration of the ion fluid into the separatrix region," J. Geophys. Res. 110, A09206, https://doi.org/10.1029/2004JA010708 (2005).

Yamada, M., Kulsrud, R., and Ji, H., "Magnetic reconnection," Rev. Mod. Phys. 82, 603-664 (2010)

Zelenyi, L. M., Lominadze, J. G., and Taktakishvili, A. L., "Generation of the energetic proton and electron bursts in planetary magnetotails," J. Geophys. Res. 95, 3883-3891, https://doi.org/10.1029/JA095iA04p03883 (1990).

Zelenyi, L. M., Artemyev, A. V., Malova, H. V., and Popov, V. Y., "Marginal stability of thin current sheets in the Earth's magnetotail," J. Atmos. Sol. Terr. Phys. 70, 325-333 (2008).

Zelenyi, L. M., Malova, H. V., Artemyev, A. V., Yu Popov, V., and Petrukovich, A. A., "Thin current sheets in collisionless plasma: Equilibrium structure, plasma instabilities, and particle acceleration," Plasma Phys. Rep. 37, 118-160 (2011).

Zhou, X. Z., Angelopoulos, V., Sergeev, V. A., and Runov, A., “Accelerated ions ahead of earthward propagating dipolarization fronts," J. Geophys. Res. 115, A00103, https://doi.org/10.1029/2010JA015481 (2010).

Zhou, X. Z., Ge, Y. S., Angelopoulos, V., Runov, A., Liang, J., Xing, X., Raeder, J., and Zong, Q. G., "Dipolarization fronts and associated auroral activities: 2. Acceleration of ions and their subsequent behavior," J. Geophys. Res. 117, A10227, https://doi.org/10.1029/2012JA017677 (2012).

Zong, Q.-G. et al., "Geotail observations of energetic ion species and magnetic field in plasmoid-like structures in the course of an isolated substorm event," J Geophys. Res. 102, 11409-11428, https://doi.org/10.1029/97JA00076 (1997). 